\title{
El análisis de patentes como estrategia para la toma de decisiones innovadoras
}

\author{
Por Maidelyn Díaz-Pérez y Félix de Moya-Anegón
}

\begin{abstract}
Resumen: La aplicación de análisis métrico a la información contenida en los documentos de patentes se ha convertido en una de las principales técnicas que permiten modelar los escenarios tecnológicos de países, empresas, institutos de investigación, proyectos, etc. La presente investigación propone la utilización de indicadores bibliométricos a las patentes, identificando patrones y tendencias a partir de la evidencia tecnológica disponible así como detectar posibles redes sociales innovadoras encubiertas, permitiendo tomar decisiones oportunas si la investigación lo requiere. Utilizamos un caso práctico para mostrar a través de sus resultados estos planteamientos, analizando la base de datos de las patentes de EUA, procesándola con una herramienta desarrollada por la Universidad de Pinar del Río, utilizando sólo ocasionalmente el software Ucinet para representar las redes sociales existentes. Esto permite llegar a resultados que demuestran la aplicabilidad de los estudios patentométricos en la ejecución de proyectos de investigación, permitiendo reorientar los objetivos de la misma.
\end{abstract}

Palabras clave: Información de patentes, Innovación, Indicadores bibliométricos, Redes sociales, Visualización de datos de patentes, Software de análisis de patentes, Aceites esenciales del Eucalyptus citriodora.

\section{Title: Patent analysis as a strategy for innovative decisionmaking}

Abstract: Applying metric analysis to the information contained in patent documentation has become one of the principal techniques for modeling technology scenarios for government, business and industry, research institutes, projects, etc. We propose using bibliometric indicators for patent research, identifying patterns and trends based on available technological evidence as well as detecting possible social networks in the "hidden web", which permits effective decisionmaking in the research context. A case study is used to demonstrate these concepts, analysing the EUA patents database and processing it with a tool developed by Pinar del Río University. Ucinet software is used on occasion to map existing social networks. This makes it possible to produce results that demonstrate the relevance of patentometric studies to research projects, including the possibility of redirecting the objectives of a research project on the basis of this analysis.

Keywords: Patent information, Innovation, Inventions, Creativity, Bibliometric indicators, Social networks, Visualization of patent data, Patent analysis software, Essential oils of Eucalyptus citriodora.

Díaz-Pérez, Maidelyn; Moya-Anegón, Félix de. "El análisis de patentes como estrategia para la toma de decisiones innovadoras”. En: El profesional de la información, 2008, mayo-junio, v. 17, n. 3, pp. 293-302.

DOI: 10.3145/epi.2008.may.05

\section{Introducción}

La llamada patentometría, o bibliometría de patentes, ha sido poco conceptualizada hasta el momento. En 2001 se encuentra en internet una definición del Insti- tuto Batelle en la $R A N D^{1}$ (contracción de los términos en ingles Research and Development), como un método de evaluación asociado con la identificación de las fortalezas y debilidades de la ciencia y la tecnología, 
a través del examen de los registros de invenciones e innovaciones provenientes de un país, institución o temática determinada.

Puede ser ocasional encontrar también la expresión bibliometría evaluativa, terminología acuñada por Francis Narin y un grupo de investigadores en Computer Horizon, Inc. (CHI) para la evaluación de la actividad científica y tecnológica. Específicamente orientada a la utilización de indicadores obtenidos mediante métodos bibliométricos, con particular énfasis en el análisis de las referencias bibliográficas que aparecen en los trabajos científicos y las patentes, para apoyar los procesos de evaluación de la ciencia. Extendido a las empresas productivas y de servicios como parte de las herramientas en la vigilancia científica y tecnológica, así como en los observatorios de ciencia y tecnología.

Estas nuevas variantes de análisis tomaron más vigor tras la aparición del Manual de patentes en 1994, en donde se recomiendan los datos de las bases de datos especializadas como indicadores de la efectividad de los medios dedicados a la innovación y del comportamiento tecnológico de empresas, industrias, áreas tecnológicas, países y regiones, etc. Y por otra parte al ser la información bibliográfica susceptible de ser cuantificada y analizada, como planteó Garfield (1978), la bibliometría se alza como disciplina idónea para el tratamiento de la información de patentes, lo que permite realizar diferentes estudios métricos.

\section{"La utilización de patentes para la toma de decisiones todavía no es un instrumento común en todos los frentes de investigación innovadores"}

Las patentes son las fuentes de información más relevantes para analizar los productos tecnológicos. Este argumento responde a la idoneidad de sus datos para su utilización estadística (Van-Leuven, 1996); a su estructura uniforme, reconocida internacionalmente; a la informatización de sus registros; y la aceptación de que por analogía las patentes son un producto básico de la actividad tecnológica como lo es el artículo en la actividad científica.

Sin embargo, aunque "las patentes representen invenciones técnicas que han pasado un examen en una Oficina, tanto para asegurar su novedad como para precisar su utilidad potencial" (Sancho) y ser considerada la información registrada en ellas como un "indicador del cambio tecnológico" (Sanz; Arias), su aplicación para la toma de decisiones todavía no es común en to- dos los frentes de investigación innovadores. Las causas suelen ser muchas pero lo cierto es que aún se carece de:

- Cultura tecnológica que permita a los investigadores utilizar los análisis de forma sostenida antes y durante todo el ciclo de vida de un proyecto de investigación (Díaz, 2005).

- Capacidad de patentar conocimientos tecnológicos relevantes obtenidos de investigaciones importantes (Díaz, 2005).

- Falta de iniciativa de las empresas, entidades, industrias, grupos de investigación, etc., para utilizar y analizar la información contenida en las patentes con una perspectiva estratégica.

Todo ello provoca una situación que incide no sólo en los resultados de la investigación sino también en algunos macroindicadores de los países. De hecho merece la pena recordar la desproporción existente entre la producción científica y la tecnológica en todo el mundo (Díaz, 2005).

Sin necesidad de invertir en recursos de información costosos es posible descubrir la novedad inesperada que puede producir un cambio, o el surgimiento abrupto de una tecnología durante una investigación, así como conocer las redes que se pueden establecer entre inventores y titulares en su generación y elaboración. Conocer las relaciones de colaboración que han propiciado un nuevo conocimiento tecnológico constituye un elemento estratégico en el proceso de vigilancia tecnológica de cualquier investigación, proyecto, negociación, etc., que se esté monitoreando. Además, resulta un estudio novedoso y poco abordado, no encontrándose muchos estudios sobre este tema en la literatura científica.

Se considera que lo que realmente debe ser analizado en los nuevos contextos de cambio, colaboración e intercambio tecnológico, son los vínculos entre las invenciones en cualquiera de sus nodos. Estamos seguros que las redes que se establecen entre los círculos de innovación y la forma de explotarlas para conseguir en el menor tiempo posible el máximo de innovación o transferencia tecnológica, debe ser una de las quimeras de este siglo en políticas de ciencia y tecnología.

En este trabajo presentamos un caso de estudio que permite mostrar los argumentos anteriores: el proyecto de composición química de la madera, la corteza y el follaje de tres especies de eucaliptos de la provincia de Pinar del Río.

\section{Métodos y procedimientos}

El proyecto se ha realizado en grupo de investigación de gran liderazgo tecnológico de la Universidad de 
Pinar del Río (UPR), Cuba. Las palabras clave que se utilizan para la búsqueda de patentes fueron: Eucalyptus citriodora, Eucalyptus saligna Smith, Eucalyptus grandis, Eucalyptus pellita. El período de tiempo cubierto es desde 1976 hasta inicios de marzo de 2005.

La fuente de información seleccionada para el estudio fue la base de datos de patentes de la US Patent \& Trademark Office (Uspto), una de las tres bases de datos más importantes del mundo (Michel; Bernd, 2001), junto a la europea y la japonesa pues entre las tres procesan el $86 \%$ de las solicitudes de patentes del mundo (Trilateral Co-operation, 2005); y por publicar íntegramente su texto (desde 1976). Además sus datos son considerados probablemente los más fiables dado el rigor y el alcance de su legislación sobre patentes, el grado de detalle y accesibilidad de los mismos, y lo atractivo de su mercado (Pavitt, 1988). Además se ha usado la base de datos de la Canadian Intellectual Property Office (CIPO) en forma menos relevante (al no encontrarse suficientes resultados), y se ha hecho una búsqueda en la Oficina Cubana de la Propiedad Industrial $(O C P I)$.

La información se procesó con Progintec ${ }^{2}$, que integra todas las etapas necesarias para el análisis de la información proveniente de bases de datos de patentes. Se trata de un programa ad hoc elaborado con PHP por el Grupo de Gestión de Información y Conocimiento de la UPR. Como gestor de base de datos se emplea postgreSQL y como servidor web Apache (ambos son software libre). Además se utilizó Ucinet para visualizar los resultados de los indicadores que muestran relaciones dentro del análisis.

Los indicadores empleados en el caso de estudio provienen de la vertiente de la escuela francesa (Centre de Sociologie de l'Innovation y el Institut de l'Information Scientifique et Technique), quienes los clasifican en indicadores de actividad, primera generación y segunda generación, etc. Se selecciona esta tendencia europea porque se considera muy práctica, abarcadora, con manuales disponibles y muy acorde con los objetivos de la presente investigación. Se determina:

- número y distribución de patentes por año de prioridad;

- productividad de invenciones por países y autores;

- capacidad de retención y captura de conocimiento en función del país de procedencia de los inventores y titulares;

- distribución de las temáticas según la Clasificación Internacional de Patentes (CIP);

- distribución de la CIP según los años de prioridad;
- inventores, signatarios y clasificaciones conjuntas.

También se consideran la co-asignación y el coword, siendo este último indicador muy significativo para los intereses de este estudio en particular. Se realiza además un análisis por temáticas en función de una clasificación ejecutada por expertos.

Todo el estudio se realiza asumiendo que "la innovación, como todo producto del desarrollo del conocimiento, es el resultado de un proceso de interacción entre un conjunto de actores que forman parte de ese mismo sistema de I+D y de innovación" (Sanz, 2001). También se consideran «las redes de innovación como el preciso espacio donde se generan, intercambian y se utilizan los conocimientos que conducen el desarrollo de la innovación" (Sanz, 2001). Es un hecho que las relaciones e interacciones que se establecen entre técnicos e investigadores favorecen el proceso de innovación.

\section{Caso de estudio}

Los productos forestales no madereros (pfnm), como alimentos silvestres, hierbas medicinales y plantas, son en su mayoría bienes de subsistencia con los que sólo se comercia en mercados locales. Sin embargo "se estima que unos 150 pfnm son objeto de comercio internacional" (Depósito de documentos de la FAO, 2001). Aunque la dependencia de muchos productos de subsistencia puede disminuir, el aumento de la demanda de alimentos y medicinas étnicos puede tener como resultado un cultivo más sistemático de algunos pfnm. El acceso a los conocimientos y tecnologías necesarias será decisivo para que las comunidades locales puedan beneficiarse de esta tendencia.

El Centro de Estudios Forestales de la UPR investiga desde la década de los noventa la biomasa forestal con el fin de proponer aprovechamientos completos del árbol, por lo que la conjunción de especialistas de diferentes perfiles ha permitido alcanzar algunos resultados en este sentido. Un ejemplo son los resultados obtenidos por los grupos de lignocelulósicos y pfnm en el "Proyecto sobre la composición química de la madera, corteza y follaje de especies de eucaliptos que crecen en la provincia de Pinar del Río" (Orea; Cordero, 1996), que generaron 7 solicitudes de patentes, convirtiéndose en el grupo de investigación con mayor liderazgo tecnológico de la $U P R$. Tales solicitudes no han sido concedidas por cuestiones burocráticas de la $O C P I$, a pesar de presentar suficientes elementos innovadores. Todo ello incide en la sostenibilidad de las investigaciones y en la posibilidad de aprobar nuevos proyectos sobre el tema a este grupo de investigación.

Los investigadores de la $U P R$ se encuentran enfrascados en un análisis exhaustivo de esta temática, dentro 
de lo cual se propuso realizar un estudio patentométrico que permita comparar y comprobar la veracidad de los resultados de las investigaciones realizadas, y determinar las proyecciones investigativas del Eucalyptus citriodora $(E C)$ para próximos proyectos. Se ha utilizado para obtener tales resultados la propuesta de aplicar indicadores bibliométricos al documento de patente, objetivo fundamental del presente estudio

\section{Análisis de los datos}

Tras analizar los documentos recuperados en la búsqueda, se decidió tratar solamente en este momento los registros que contenían los términos Eucalyptus citriodora $(E C)$ en cualquier campo de la patente, dada la importancia que en la literatura tiene esta especie en particular. De un total de 224 invenciones recuperadas se procesaron por el sistema solamente 81 registros. La poda se estableció en función de diferentes criterios colegiados con los expertos, por ejemplo se separaron las patentes que reivindicaban procedimientos de plantación, aprovechamiento de otras partes del árbol, mejoramiento genético de la planta, etc., dejando solamente las 81 patentes que se relacionan con los fines de investigación del proyecto que se analiza.

El primer indicador aplicado fue el de actividad, mostrándose el número de invenciones por fecha de prioridad (gráfico 1). Se observa que es una tecnología nueva (la primera patente sobre la temática data de 1989), pero no es hasta 1999 que comienza a crecer el promedio por año, mostrándose los más recientes como

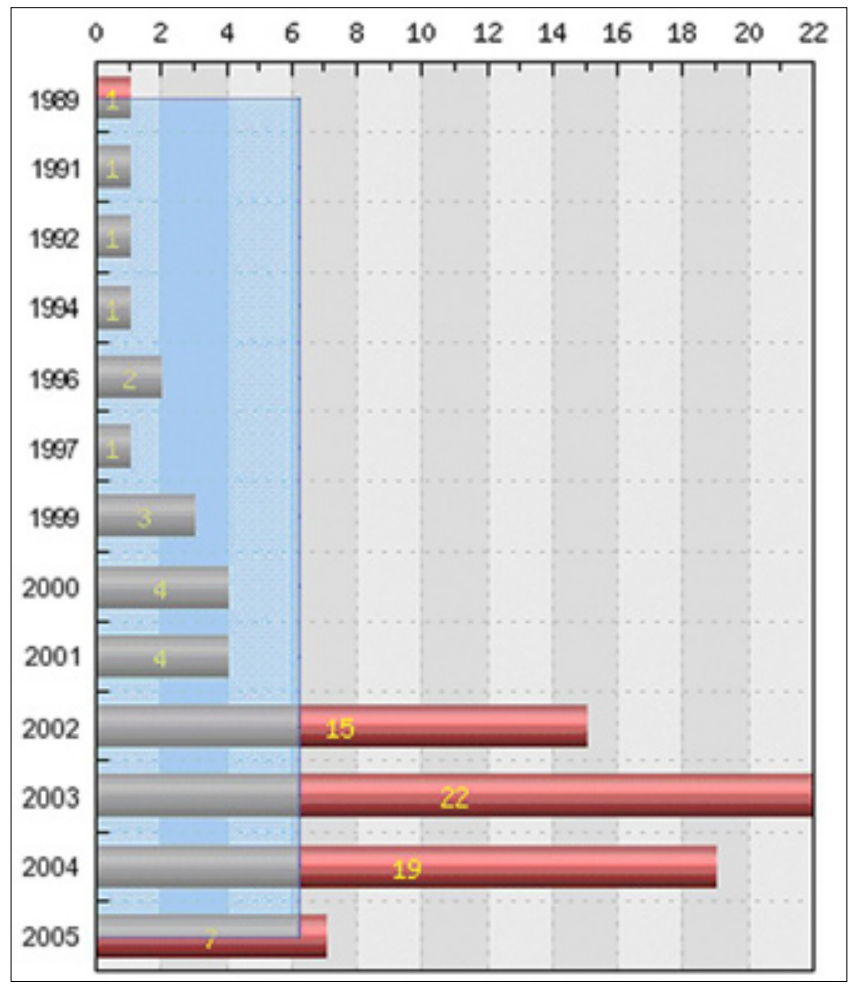

Grafico 1. Número de invenciones por fecha de prioridad los de mayor actividad inventiva, lo que revela el interés reciente en investigar el $E C$ y protegerlo legalmente en este continente.

Al analizar la procedencia de estas invenciones por país del primer inventor y cantidad de inventores por países, se observa que el frente de investigación de esta temática está compuesto por un grupo reducido de países donde se origina la tecnología con un número limitado de investigadores, apareciendo un grupo de ellos con mayor presencia en EUA, Alemania, Japón y Australia que trabajan juntos en diferentes años (figura 1). Un elemento significativo del estudio es que a pesar de que existen varios inventores de un mismo país trabajando en una misma temática y año, no se muestran relaciones de coautoría.

Los líderes en invenciones son: John G. Babish, de EUA (6 patentes); Jorg-Peter Schur de Alemania (5); y Marcus Eh también de Alemania (4), todos colocados en el centro del gráfico por tener invenciones concedidas en varios años. Uno de los nodos aislados es el autor de la primera patente (1989) que no parece haber vuelto a generar nada más, al menos con ese nombre y en esa oficina.

Otro elemento significativo es el escaso nivel de productividad científica medida en términos de patentes concedidas y solicitadas que presentan las universidades. No se registraron patentes en las que se hiciera evidente la colaboración entre universidades y organizaciones privadas o de otro tipo, a pesar de que la Universidad de Michigan es una de las propietarias de la tecnología.

Aunque no existe coautoría, para nuestra sorpresa sí se presenta una ligera titulación conjunta (figura 2) entre determinados propietarios e inventores con permanencia en la temática, lo que pudiera significar en un futuro, posibles coautorías u alianzas estratégicas. La mayoría de las invenciones pertenecen a EUA, no mostrándose titulación conjunta entre las firmas propietarias. Esa deber ser la estrategia de patentamiento de las grandes empresas reconocidas en el mercado como $\mathrm{Ba}$ yer y Lion Corporation, argumentos que habitualmente son válidos en los sectores de alta tecnología.

Los estudios sobre "el origen y el destino de las invenciones son de imprescindible importancia a la hora de analizar los indicadores de capacidad de retención ${ }^{3}$ y captura de conocimiento " (Maspons; Escorsa) de un determinado dominio tecnológico. Es una de las formas de medir su capacidad de innovación y cambio tecnológico, pero también su potencial de integración en redes innovadoras.

En los análisis anteriores los EUA se presentan como el país con mayor porcentaje de investigadores, lo que se corresponde con los derechos de explotación 


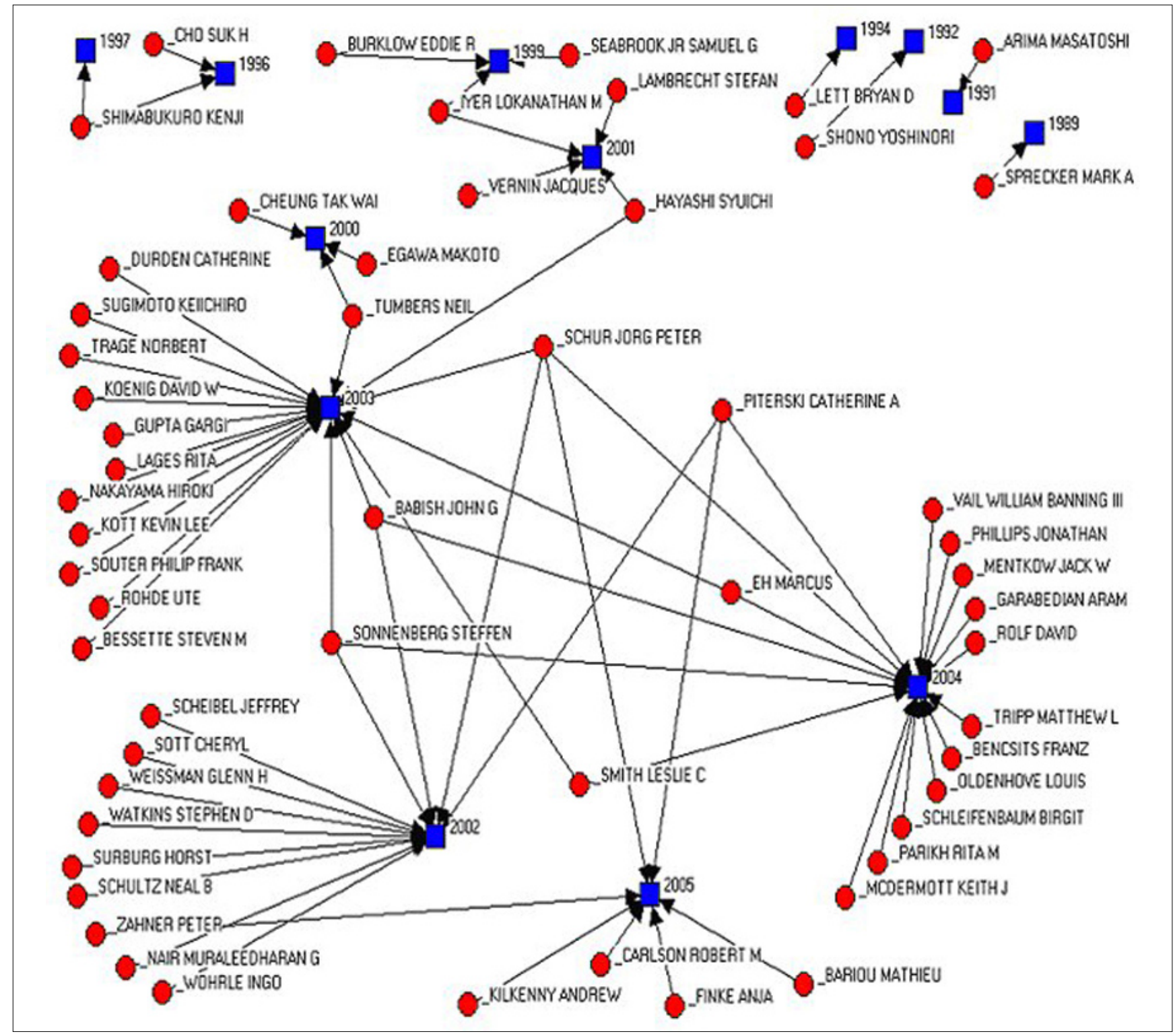

Figura 1. Comportamiento de inventores por año. Fuente: Ucinet

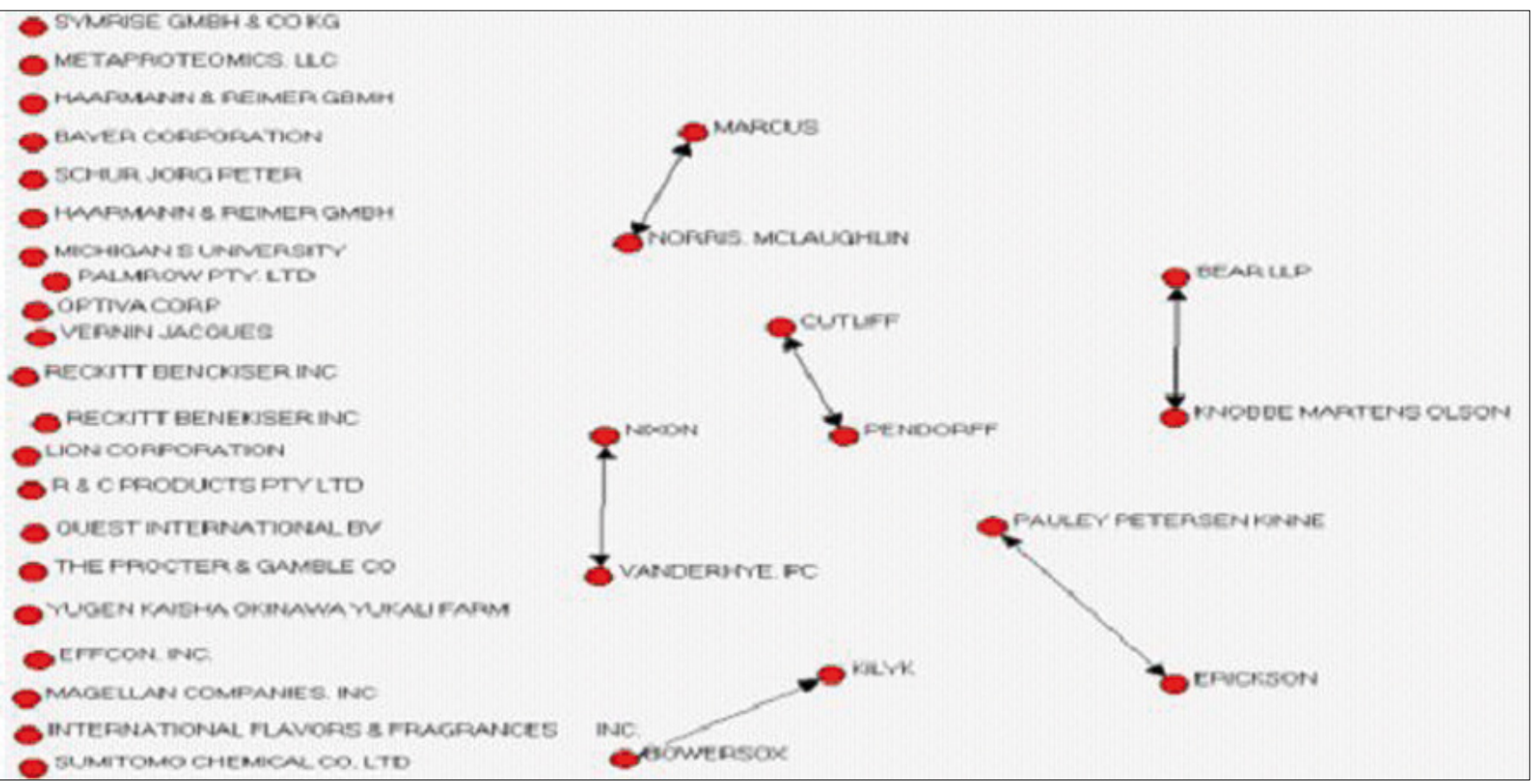

Figura 2. Comportamiento de los titulares (co-asignación). Fuente: Ucinet 
de las patentes. Este comportamiento indica una fuerte retención de conocimiento de una tecnología y un mercado bien establecido en dicho sector técnico, así como una marcada capacidad para la captura de conocimiento y tecnología foránea. Por ejemplo países fuertes como Alemania y Japón originan tecnologías que son comercializadas por otros países como EUA (a través de 47 titulares), lo que se explica por la gran potencia de la industria y el mercado en esa nación. Y si analizamos este comportamiento desde la posición de los restantes países, observamos una migración o fuga de conocimiento técnico. Algunos países generan invenciones que luego son explotadas en otros.

"Un grupo de países generan invenciones que son protegidas en forma de patentes de invención en otros países que no son las que las originan"

Obtenida una visión más práctica de cuándo, dónde y quiénes producen las tecnologías basadas en el $E C$, a

\section{Co-citas}

La co-citación bibliográfica permite establecer una similitud temática entre dos documentos. Si A y B son citados por C, puede decirse que ambos se relacionan entre sí, aunque ellos no se refieran directamente del uno al otro. Si $A$ y $B$ son citados a la vez por muchos otros documentos, se podrá deducir que tienen una relación mucho más fuerte entre sí. Cuantas más citas simultáneas reciban, más probabilidad existe de que traten el mismo tema.

continuación se prosigue con un análisis más orientado a los contenidos de las patentes y sus relaciones.

La figura 3 muestra, del total de patentes, las más co-citadas, parejas de invenciones que resultan ser las más consultadas para generar nuevos resultados investigativos en esta temática. Destaca la pareja US5549840-

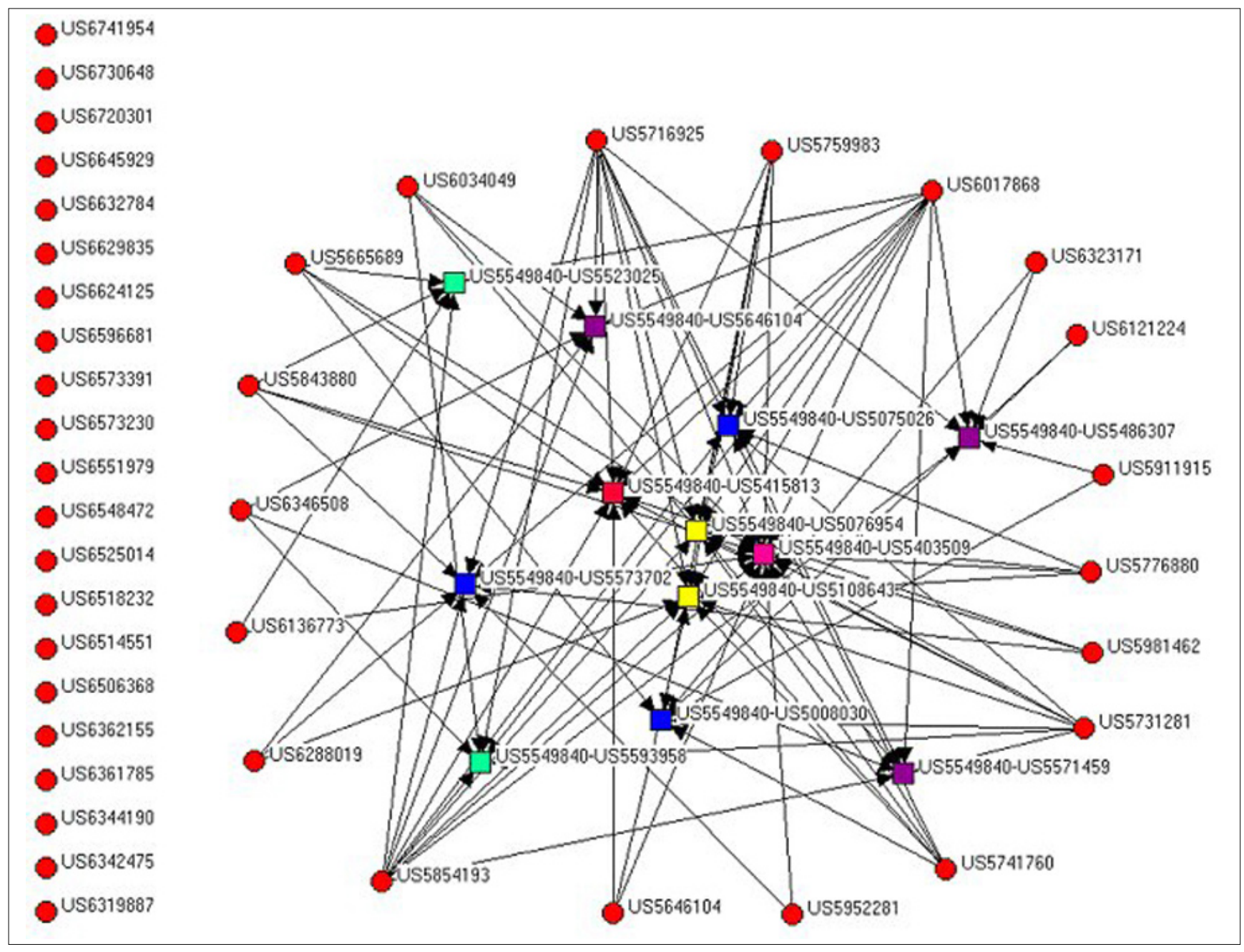

Figura 3. Patentes más cocitadas. Fuente: Ucinet 
US5403509 como las más cocitadas 5 . Colocadas en el centro, y con diferentes colores correspondientes a la cantidad de veces que fueron nombradas, se encuentran las cocitaciones. Son invenciones que por su gran co-ocurrencia en las referencias deben formar parte del frente de investigación tecnológico y del estado de la técnica de la temática objeto de estudio. Y dentro de ellas la patente más citada es la US5549840, considerada por los investigadores de la UPR como una tecnología fundacional.

Desde otra posición, la figura 4 muestra las patentes que más citan estas parejas de invenciones, mostrando las que más dependencia tecnológica tienen de estas cocitaciones para generar nuevas invenciones. De este comportamiento se puede inferir por ejemplo que existe copia de tecnología o relaciones de colaboración e intercambio entre sus tecnólogos, etc. Otra observación que se desprende del análisis de cocitación es que su estudio representa una evaluación de la tecnología generada, algo muy diferente a lo que sucede en la cocitación de la literatura no-patente. En el contexto tecnológico "la cita es una consulta obligada en la revisión del estado de la técnica, una garantía de calidad de la invención, transparencia que sabemos muy difícil de distinguir en las citaciones de artículos científicos" (Díaz, 2007).

Las patentes se agruparon por clases de la Clasificación Internacional de Patentes (CIP), lo que permitió observar la especialización del conocimiento inventivo por sectores tecnológicos. En este caso se encontraron 2 clases más representadas:

- C11D: detergent compositions (preparations specially adapted for washing the hair; methods or apparatus for disinfection or sterilisation; special washing compositions for cleaning semi-permeable membranes; use of single substances as detergents; soap or soap-making; resin soaps; recovery of glycerol.

- A61K: preparations for medical, dental, or toilet purposes (bringing into special physical form; chemical aspects of, or use of materials for deodorisation of air, for disinfection or sterilisation, or for bandages, dressings, absorbent pads or surgical articles; compounds per se C01, C07, C08, C12N; soap compositions C11D; micro-organisms per se C12N).

Respecto a esto, una de las redes más representativas para el estudio es la que muestra la figura 5 . La primera patente concedida está orientada a la CIP

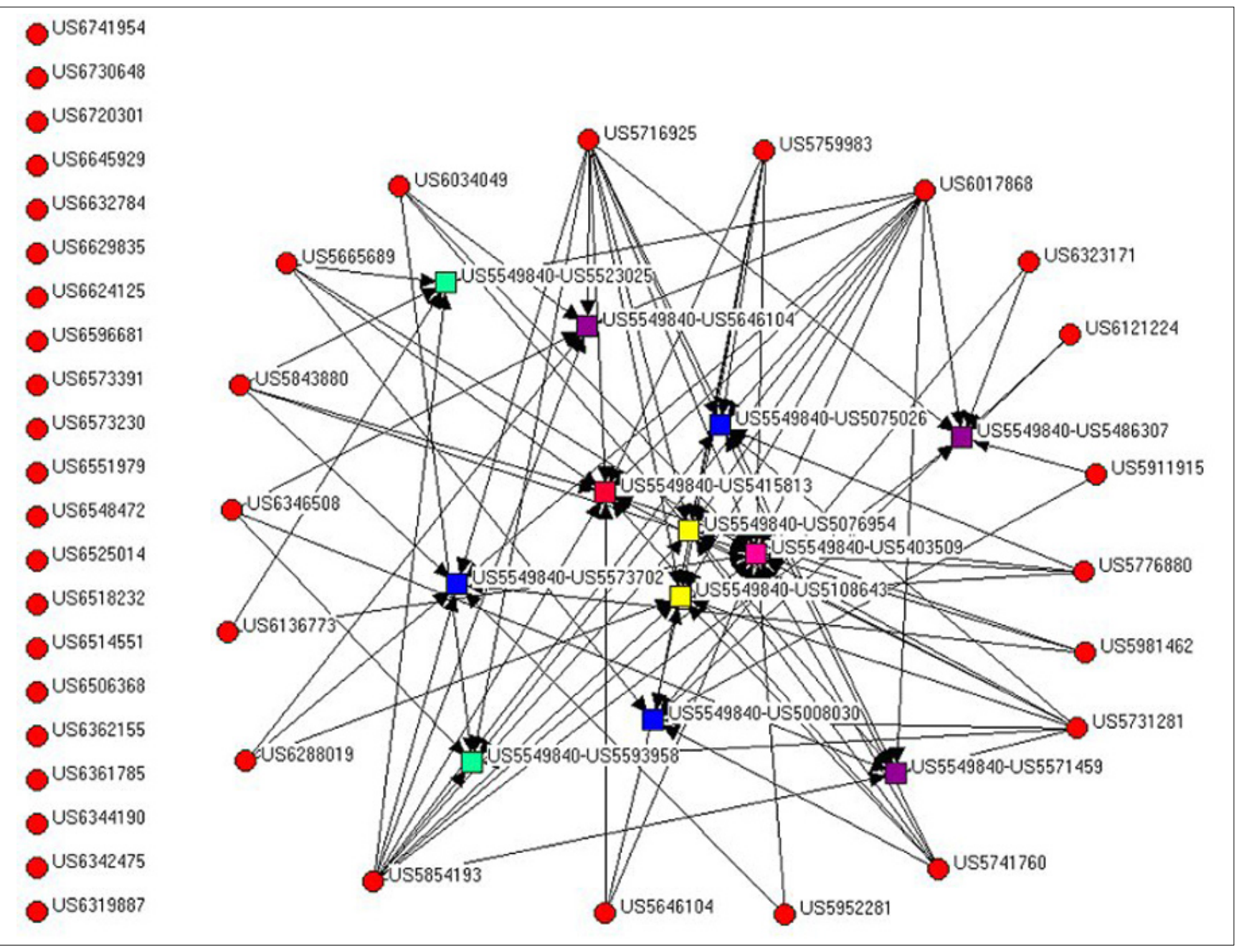

Figura 4. Patentes con más dependencia tecnológica. Fuente: Ucine 


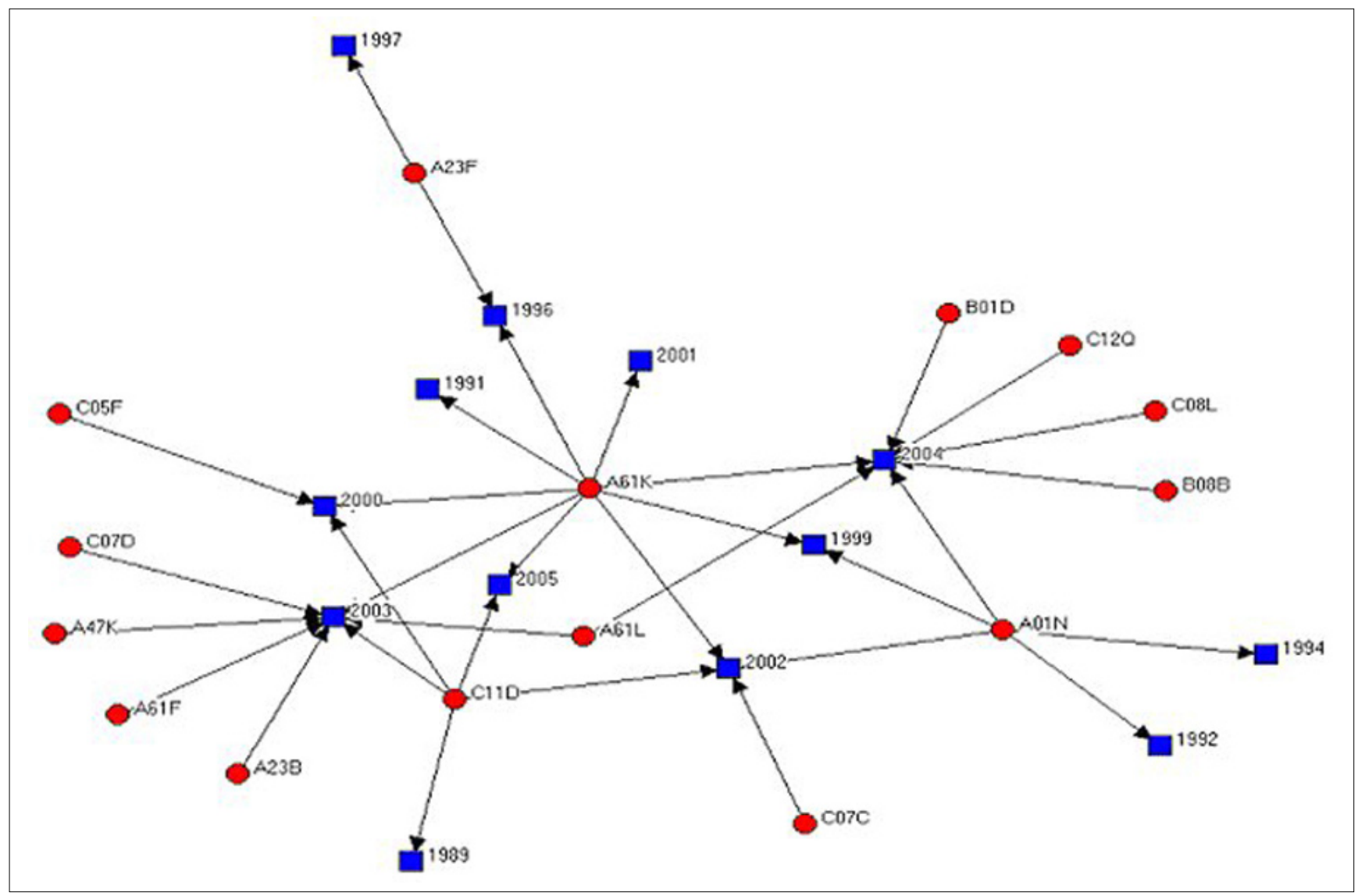

Figura 5. Comportamiento de la CIP por año. Fuente: Ucinet

C11D pero sin embargo el nodo central, con más vínculos desde el año 1991 y con mayor sostenibilidad en el período de tiempo estudiado, es la $C I P A 61 K$, la cual presenta también relaciones con otros nudos temáticos que en estos momentos están bajo estudio por parte de los investigadores de la $U P R$.

La aplicación del indicador co-word en algunos campos importantes de la patente permitió reducir su texto a un número de términos con significado que ocurren y co-ocurren $\mathrm{X}$ veces (figura 6 ).

La categoría que muestra más centralidad es oil (aceites), lo que se corresponde con el uso del $E C$ en este estudio, en este caso los extract essential oil (extractos de aceites esenciales). Se colocan las tres categorías temáticas más importantes en el centro del gráfico, sirviendo a su vez de nodos de intermediación a las restantes. Todos los grupos que se destacan están en función de sus aplicaciones: aceites para insecticidas y repelentes (incluyéndose átomos de carbono, hidrógeno, sodio..., datos importantes para los especialistas); productos de fragancia, relacionados con los extractos de aceite de pino; extractos de aceites esenciales con fines farmacológicos -utilizados como anti-inflamatorios, etc.-. Se observó una plena correspondencia entre el análisis de co-ocurrencia de palabras y el realizado con la $C I P$.
Los investigadores también realizaron agrupaciones temáticas manuales, lo que permitió ajustar los resultados de tendencias a los objetivos concretos del proyecto. Así, se comprueba que la mayor parte de las invenciones utiliza el aceite del $E C$ y otras utilizan el extracto. El mayor número de invenciones se concentra en productos vinculados a medicamentos, y también otras de cosmética, fertilizantes, insecticidas, etc.

\section{Principales resultados}

El proceso, análisis y visualización de la información contenida en los registros de invenciones facilitó la toma de decisiones innovadoras de los investigadores y de la Vicerrectoría de Investigaciones de la UPR. Se pudieron reorientar a tiempo los objetivos de las investigaciones proyectadas, ofreciendo la oportunidad de elaborar inmediatamente dos nuevas propuestas de solicitudes de invención correctamente respaldas por el estado de la técnica ${ }^{6}$.

Los resultados de los análisis junto a las descripciones técnicas, permitieron a los investigadores líderes del proyecto:

- Encaminar nuevas investigaciones sobre evaluación toxicológica y preclínica de los extractos de follaje y corteza de las especies estudiadas con el fin de ser utilizadas en la obtención de nuevos fármacos. 


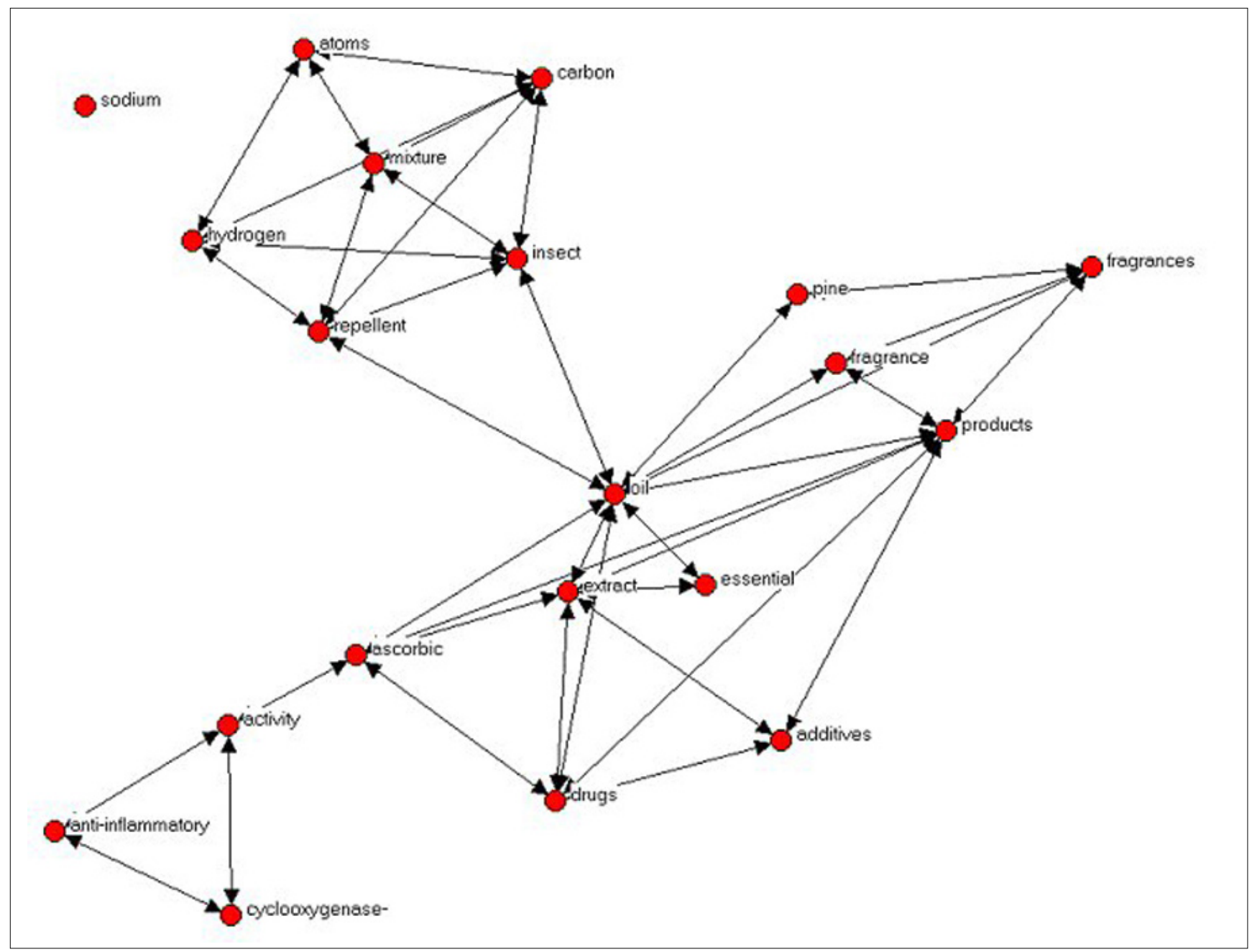

Figura 6. Coocurrencia de palabras con significado. Fuente: Ucinet

- Orientar las investigaciones especialmente a la oftalmología y la dermatología, presentando recientemente el proyecto: Evaluación toxicológica y preclínica de extractos de plantas con fines oftalmológicos y dérmicos.

\section{"Los resultados confirman la importancia de aplicar indicadores bibliométricos a la documentación de patentes"}

- Disponer de respaldo técnico y bibliográfico para obtener nuevos productos farmacológicos de circulación nacional como: antifúngicos, antibacterianos, anti-inflamatorios y especialmente uno (aún bajo estudio) para combatir la obesidad.

- Inferir que pueden existir derivados de clorofila, a partir de residuos forestales y no únicamente de algas y otras especies marinas, resultado que está en fase de elaboración y que será presentado próximamente en un evento sobre la temática en Italia.
- Saber que el país con mayor actividad tecnológica y líder en innovación (medido a partir de las bases de datos estudiadas) es EUA, seguido de Alemania y Japón.

- Conocer que el número creciente de solicitudes en el período 2000-2005 demuestra que la especie EC constituye un producto natural de interés para el mundo científico tecnológico.

- Reorientar los fines de utilización de las investigaciones antes del análisis, orientado al estudio de la composición química de la corteza y el follaje desde el punto de vista forestal, ahora encaminado a las industrias farmacéutica, cosmética, además de la forestal y agronómica, lo que muestra un incremento en la cobertura temática.

- Redireccionar las investigaciones de eucalyptus de las distintas especies, en madera, corteza, hojas y composición química a: aceites esenciales, antioxidantes, antifúngicos, insecticidas, cosmética, etc. Y en un segundo momento, reorientar el estudio del objeto forestal: el árbol, la corteza, el follaje, a los efectos de los extractos de estas distintas partes del árbol, en animales y humanos. 
Todos ellos son resultados que confirman la propuesta y la importancia de uso y aplicación de indicadores bibliométricos al documento de patente, con vistas a identificar, a través de diferentes análisis métricos y relacionales, los comportamientos que se pueden presentar y sean de interés ante cualquier toma de decisión innovadora durante una investigación, proyecto, etc.

\section{Notas}

1. Rand emplea a más de 600 profesionales de la investigación, el $80 \%$ de los cuales son doctores. La sede central está en Santa Mónica, California.

2. Grupo coordinado por Maidelyn Díaz-Pérez, una de las autoras de esta investigación.

3. Capacidad de retención se aplica a los países que generan y a su vez explotan la tecnología.

4. Por captura de conocimiento se entiende en este estudio los países que no figuran en el campo inventores y aparecen como titulares de la invención generada.

5. Por limitaciones de la base de datos utilizada no puede realizarse en este caso específico la extracción automática de los datos (autores, titulares, $C I P$ ) de las patentes co-citadas, dificultando su visualización y representación. En otros estudios sí es posible visualizar las parejas de inventores, las CIP y signatarios.

6. La necesidad de proteger los intereses del mismo no permite declarar abiertamente todos los resultados del presente análisis, razón por la que únicamente mostraremos algunas de las principales contribuciones.

\section{Bibliografía}

Depósitos de documentos de la FAO. Hacia una silvicultura sostenible. Agricultura Mundial hacia los años 2015-2030. Departamento Económico y Social. Isaaa, 2001.

Díaz, M. La información de patentes en el ciclo de vida de un proyecto de investigación: caso de estudio [Tesis en opción al título de Master en ciencias de la información]. Facultad de Comunicación e Información Científica, La Habana, 2005.

Díaz, M. "La información de patentes en la gestión prospectiva en salud". En: $6^{\circ}$ Congreso internacional de informática en salud, 2007.
Garfield, E., et al. Citation data as science indicators. Toward a metric of science. New York: Wiley, 1978.

Maspons, R.; Escorsa, P. Los flujos de conocimiento hacia Barcelona y desde Barcelona: análisis mediante la información contenida en las patentes. IALE Tecnología.

Michel, J.; Bernd, B. «Patent citation analysis: a closer look at the basic input data from patent search reports». En: Scientometrics, 2001, v. 51, n. 1, pp. 185-201.

Orea, U.; Cordero, E. Caracterización química de la madera, corteza y follaje de tres especies de eucaliptos de la provincia de Pinar del Río, Cuba. Proyecto de investigación, 1996.

Pavitt, K. "Uses and abuses of patent statistics". En: Van Raan, A. F. J. (ed.). Handbook of quantitative studies of science and technology. Elsevier Science Publishers B. V., North Holland, 1988.

Sancho, R. Indicadores de los sistemas de ciencia, tecnología e innovación. Ministerio de Ciencia y Tecnología, España.

Sanz, L. Indicadores relacionales y redes sociales en el estudio de los efectos de las políticas de ciencia y tecnología. Spanish Policy Research in Innovation \& Tecnology, Training, Education, 2001.

Sanz, L.; Arias, E. Especialización y capacidades tecnológicas de las regiones españolas: un análisis a través de patentes europeas. Instituto de Estudios Sociales Avanzados (CSIC).

Trilateral co-operation. Trilateral statistical report 2004. Edition Munich, Germany, 2005.

http://www.trilateral.net/tsr/tsr_2004/ch2/

Van-Leuven, J. W. Patent statistics as indicator for innovation. Patent Word, 1996.

Maidelyn Díaz-Pérez, coordinadora del Departamento de Gestión de Información y Conocimiento y profesor asistente de la Universidad de Pinar del Río, Cuba. mdp73@correo.ugr.es

Félix de Moya-Anegón, vicerrector de Investigaciones de Postgrado y Profesor Titular en la Universidad de Granada.

felix@ugr.es

\section{Quieres recibir tu copia del Anuario ThinkEPI 2008 en casa*?}

